\title{
Analgesia mediated by stress: Possible antalgic mechanism of the dry needling technique
}

\author{
Pecos Martín D. ${ }^{1}$ Lázaro Navas I. ${ }^{1} \quad$ Lorenzo Sánchez-Aguilera C. ${ }^{1}$ Gallego Izquierdo T. ${ }^{1}$ \\ 1 Universidad de Alcalá, Madrid, Spain
}

Rev Fisioter Invasiva 2019;2:123.

\begin{abstract}
Keywords

- dry needling

- autonomic nervous system

- cortisol hormone

Introduction and Aim One of the possible mechanisms of action of dry needling is stress-induced analgesia, produced by the activation of the sympathetic nervous system (SNS). Cortisol is a steroid hormone, which is considered to be the most representative indicator of physiological states that are altered after a stressful stimulus. Although studies of other invasive techniques exist which measure the cortisol levels, no studies on dry needling have been found which gather this stress indicator. Thus, the aim of this study was to determine the changes produced in the levels of cortisol concentration in saliva after the application of the dry needling technique.

Material and Methods Sixty-two healthy volunteer subjects participated in this randomized controlled single blinded trial performed at the University of Alcalá. Participants were randomly distributed into two intervention groups: deep dry needling ( $n=33)$ and placebo needling (control) $(n=32)$. The saliva samples were gathered prior to the intervention and 3 minutes after finalization of the same. The level of cortisol in saliva was determined in a laboratory by performing a solid phase enzyme immunoassay using the ELISA $®$ kit. Statistical significance was set at $p<0.05$. The differences between both groups were compared based on the percentage of change between the initial level and at three minutes via the Student's $t$-test for independent samples, estimating the effect size via the Cohen's $d$ test.

Results An increase of the levels of cortisol in saliva of $11.27 \% \pm 4.76$ was observed after the deep dry needling; in the group of placebo needling, an increase of $1.51 \% \pm 3.08$ was observed. However, when comparing both groups, no statistically significant differences were obtained $(p=0.920)$, with an effect size of $d=0.42$.

Conclusions The dry needling technique leads to an increase in the release of cortisol, one of the hormones involved in the sympathetic-excitatory relationship. This could be related to one of the possible effects of this invasive procedure with mechanisms of stress-induced analgesia. However, further studies are required to deepen our knowledge on the plausible physiological effects which support the application of the dry needling technique.
\end{abstract}

